\title{
Round Robin CPU Scheduling Using Dynamic Time Quantum with Multiple Queue
}

\author{
Sonagara Payal T. \\ ${ }^{1}$ Gujarat Technology University, Shree Pandit Nathulalji Vyas Technical Campus, Wadhwan city, India
}

\begin{abstract}
Scheduling is the central concept used in operating system. It is help to choosing the processes for execution. There are many scheduling algorithms available in operating system like SJF, Priority, FCFS, Round Robin, multilevel queue etc. We mainly focused on Round Robin and multilevel queue scheduling algorithm. Performance of Round Robin algorithm depends upon the size of Fixed or Static Time Quantum (TQ). If TQ is very large then Round Robin algorithm approximate to First Come First Served. If Time Quantum is too small then there will be many context switching between the processes. If we use only FCFS then turnaround time is unpredictable and waiting time is large. To solve these types of problem we proposed a new algorithm as titled "Round Robin CPU Scheduling using Dynamic Time Quantum with Multiple Queue".
\end{abstract}

Keywords: Scheduling, Waiting Time, Context Switch, Turnaround Time.

\section{Introduction}

Operating system is one type program that controls the execution of application programs and it"s also interface between the user of a computer and the computer hardware [8]. Operating system provides a platform in which user can interact with hardware and execute programs in an efficient manner. Operating system provides various types of services like program execution, I/O operation, file system manipulation, communication and error detection [2]. Modern operating system and time sharing system are more complex, they have involved from multitasking environment in which processes run in synchronized manner [5]. Multiprogramming and multitasking operating system allows more than one process to be loaded into the executable memory at a time and for the loaded process to share the $\mathrm{CPU}$ using time multiplexing. The scheduling mechanism is the part of the process manager that handles the removal of the running process from the $\mathrm{CPU}$ and the selection of another process on the basis of a particular strategy. Selection of processes is done by the "scheduler" [6]. Many parameter effect the scheduling process such as,

\subsection{CPU Utilization}

This is measure of how much busy the CPU is. Usually, the goal is to maximize the CPU utilization. Actual CPU utilization varies depending on the amount and type of managed computing tasks [6].

\subsection{Throughput}

Number of processes that complete their execution per time unit. Throughput is defined as the amount of information passed put through or delivered in a specific period of time [6].

\subsection{Waiting time}

Amount of time a process has been waiting in the ready queue [1]. It is the time between start time and read time. Usually, the goal is to minimize the waiting time.

\subsection{Response time}

Amount of time it takes from when a request was submitted until the first response is produced that means time when task is submitted until the first response is received [5].

\subsection{Turnaround time}

Amount of time to execute a particular process. In other words it is the mean time from submission to completion of a process.

\subsection{Fairness}

In the absence of user or system supplied criteria for selection, the scheduler should allocate all process must be given equal opportunity to execute [6].

\section{Background Work}

RR scheduling algorithm working with the many strategy. We discuss different strategy working with dynamic time quantum.

\subsection{Round Robin Strategy}

In this strategy Round Robin scheduling algorithm working with static time quantum. In Round Robin scheduling algorithm the time quantum is fixed and this time quantum given to every process.

\subsection{Min Max Round Robin Strategy}

In this strategy, RR scheduling algorithm working with Dynamic Time Quantum rather than Static Time Quantum. In this technique time quantum is a difference between maximum and minimum burst time of processes. This time quantum taken as a dynamic time quantum and applying to each process. 


\section{International Journal of Science and Research (IJSR) \\ ISSN (Online): 2319-7064}

Index Copernicus Value (2013): 6.14 | Impact Factor (2014): 5.611

\subsection{Efficient Round Robin Strategy}

In this strategy, RR scheduling algorithm working with Dynamic Time Quantum rather than Static Time Quantum. In this technique first find out the median and mean value of CPU Burst time of all the processes .then we compare mean and median value. Among them greater value multiply with highest burst time and least value multiply with lowest burst time and then we find square root of it. This value taken as a Dynamic time quantum and apply to each processes.

\subsection{Average Mid Max Round Robin Strategy}

In this strategy, RR scheduling algorithm working with Dynamic Time Quantum rather than Static Time Quantum. In this technique first we find out mid value of burst time. Dynamic time quantum is the average of the summation of mid and max process. This time quantum applies each process [4].

\section{Mathematical calculation}

The number of processes and CPU Burst Time (BT) are accepted as input and Average Waiting Time (AWT), Average Turnaround Time (ATT), and number of Context Switch (CS) are produced as output. Let's consider five process $(\mathrm{P} 1, \mathrm{P} 2, \mathrm{P} 3, \mathrm{P} 4, \mathrm{P} 5)$ with arrival time $=0$ and burst time $(8,40,72,84,100)$ as shown in table 1 .

\begin{tabular}{|c|c|c|}
\hline Process & Arrival times & burst time \\
\hline P1 & 0 & 10 \\
\hline P2 & 0 & 11 \\
\hline P3 & 0 & 12 \\
\hline P4 & 0 & 13 \\
\hline P5 & 0 & 14 \\
\hline
\end{tabular}

Table 1: Process with burst time

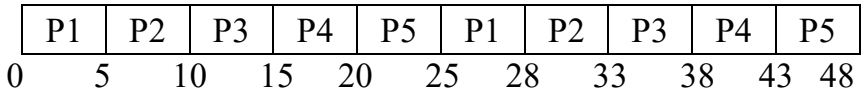

\begin{tabular}{|l|l|l|l|l|l|l|l|l|l|l|}
\hline P2 & P3 & P4 & P5 & P2 & P3 & P4 & P5 & P2 & P3 \\
\hline 48 & 53 & 58 & 63 & 68 & 73 & 78 & 83 & 88 & 93 & 98
\end{tabular}

\begin{tabular}{|l|l|l|l|l|l|l|l|l|l|}
\hline P4 & P5 & P2 & P3 & P4 & P5 & P2 & P3 & P4 & P5 \\
\hline
\end{tabular}
$\begin{array}{lllllllllll}98 & 103 & 108 & 113 & 118 & 123 & 128 & 133 & 138 & 143 & 148\end{array}$

\begin{tabular}{|l|l|l|l|l|l|l|l|l|l|l|}
\hline P2 & P3 & P4 & P5 & P3 & P4 & P5 & P3 & P4 & P5 \\
\hline 148 & 153 & 158 & 163 & 168 & 173 & 178 & 183 & 188 & 193 & 198
\end{tabular}

\begin{tabular}{|l|c|c|c|c|c|c|c|c|c|}
\hline P3 & P4 & P5 & P3 & P4 & P5 & P3 & P4 & P5 & P3 \\
\hline
\end{tabular}

\begin{tabular}{|l|l|l|l|l|l|l|l|l|l|l|}
\hline P4 & P5 & P3 & P4 & P5 & P4 & P5 & P4 & P5 & \\
\hline 248 & 253 & 258 & 260 & 265 & 270 & 275 & 280 & 284 & 304
\end{tabular}

Figure 1: Gantt chart RR Scheduling

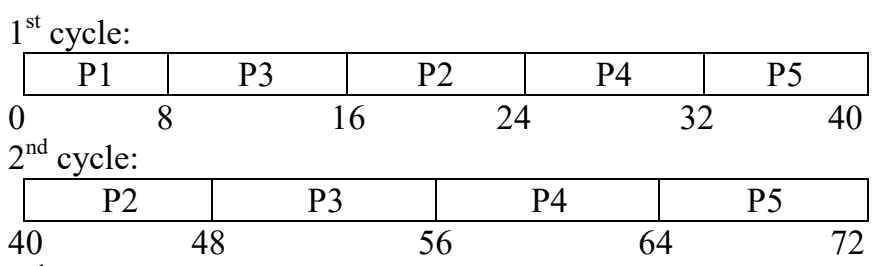

$3^{\text {rd }}$ cycle:

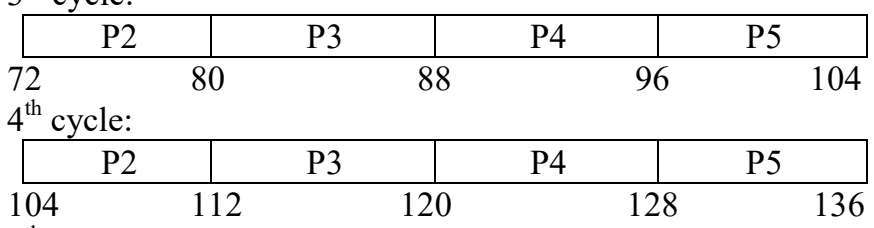

$5^{\text {th }}$ cycle:

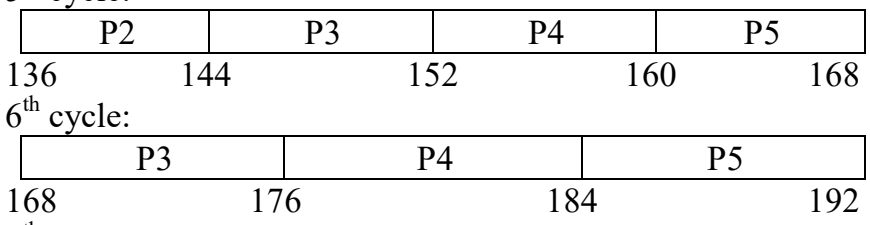

$7^{\text {th }}$ cycle:

\begin{tabular}{|ll|ll|ll|}
\hline \multicolumn{1}{|r|}{ P3 } & P4 & \multicolumn{2}{c|}{ P5 } \\
\hline \multicolumn{3}{|c|}{200} & \multicolumn{2}{c|}{208} & 216
\end{tabular}

$8^{\text {th }}$ cycle:

\begin{tabular}{|ll|ll|lll|}
\hline \multicolumn{2}{|c|}{ P3 } & & P4 & \multicolumn{2}{c|}{ P5 } \\
\hline 216 & & 224 & & 232 & & 240
\end{tabular}

$9^{\text {th }}$ cycle:

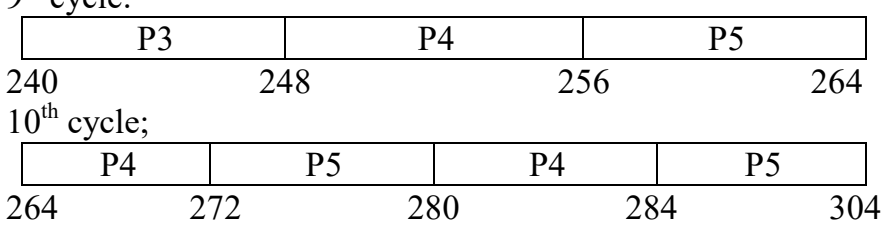

Figure 1: Gantt chart MIRR Scheduling

$1^{\text {st }}$ cycle;

\begin{tabular}{|ll|ll|lll|}
\hline \multicolumn{3}{|c|}{ P1 } & \multicolumn{3}{c|}{ P2 } \\
\hline 0 & & 8 & & & 32 \\
\hline & P3 & & P4 & & P5 & \\
\hline 32 & & 104 & & 188 & & 273 \\
\hline
\end{tabular}

$2^{\text {nd }}$ cycle:

273

P2

$3^{\text {rd }}$ cycle:

289 P5 304

Figure 1: Gantt chart RRDM Scheduling

\section{Comparison and result analysis}

\begin{tabular}{|c|c|c|c|c|}
\hline Paper & $\begin{array}{c}\text { Time } \\
\text { Quantum }\end{array}$ & ATT & AWT & CS \\
\hline RR & 5 & 206 & 145 & 58 \\
\hline MIRR & 8 & 197.6 & 136 & 36 \\
\hline RRDM & 24,85 & 178.6 & 117.8 & 7 \\
\hline
\end{tabular}

\section{Conclusion}

We have studied different multilevel queue and round robin scheduling algorithm which contain different method for find the time quantum. We have studied multilevel queue scheduling algorithm in this is use of round robin scheduling algorithm and shorted job first scheduling algorithm. This all

\section{Volume 4 Issue 11, November 2015}


algorithm studies and final we are make "round robin cpu scheduling using dynamic time quantum with multiple queue ${ }^{\text {eec. }}$. Its algorithm gives better output compare to round robin algorithm. Its efficient to the another algorithm.

\section{References}

[1] Vaishali Chahar, Supriya Raheja, Fuzzy Based Multilevel Queue Scheduling Algorithm, 2013 International Conference on Advances in Computing, Communications and Informatics.

[2] R.N.D.S.S.Kiran,Ch. Srinivasa Rao, Manchem Srinivasa Rao, Mean Interleaved Round Robin Algorithm: A Novel CPU Scheduling Algorithm for Time Sharing Systems, (C) Springer International Publishing Switzerland 2015.

[3] Vaishali Chahar, Supriya Raheja, A Review of Multilevel Queue and Multilevel Feedback Queue Scheduling Techniques, International Journal of Advanced Research in Computer science and Engineering. Vol 3, Issue 2, JUN 2013.

[4] M. V. Panduranga Rao, K. C. Shet, Analysis of New Multilevel Feedback Queue Scheduler for Real Time Kernel, International Journal of Computational Cognition.Vol. 8, No. 3, September 2010.

[5] Saroj Hiranwal, Dr. K.C. Roy, Adaptive Round Robin Scheduling using Shortest Burst Approach Based on Smart Time Slice, International Journal of Computer Science and Communication.VOL 2, No 2, July- Dec 2011.

[6] Prof Rakesh Mohanty, Prof H .S. behera, Debashree Nayak, A New Proposed Dynamic Quantum with ReAdjusted Round Robin Scheduling Algorithm and Its Performance Analysis. International Journal of computer application. VOL 5, AUGUEST 2010

\section{Author Profile}

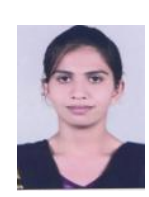

Payal Sonagara received the B.E. degrees in Computer Engineering from C. U. Shah College of Engineering and Technology in 2012. 